\title{
Mapping the contours of an emerging phase out science
}

Gregory Trencher ( $\nabla$ trencher.gregory.2s@kyoto-u.ac.jp )

Kyoto University https://orcid.org/0000-0001-8130-9146

Adrian Rinscheid

University of St. Gallen https://orcid.org/0000-0003-3160-538X

Daniel Rosenbloom

University of Toronto

Florentine Koppenborg

Technical University of Munich

Nhi Truong

Tohoku University

Pinar Temocin

University of Hiroshima

\section{Article}

Keywords: Phase-out, socio-technical transition, decline policy, technology, sustainability, environment

Posted Date: March 7th, 2022

DOI: https://doi.org/10.21203/rs.3.rs-1348121/v1

License: (c) (i) This work is licensed under a Creative Commons Attribution 4.0 International License.

Read Full License 


\title{
Mapping the contours of an emerging phase out science
}

\author{
Gregory Trencher ${ }^{1}$, Adrian Rinscheid ${ }^{2}$, Daniel Rosenbloom ${ }^{3}$, Florentine \\ Koppenborg ${ }^{4}$, Nhi Truong 5 , Pinar Temocin ${ }^{6}$ \\ 1. Kyoto University, Graduate School of Environmental Studies, Kyoto, Japan \\ Email: trencher.gregory.2s@kyoto-u.ac.jp \\ 2. University of St. Gallen, Institute for Economy and the Environment, Switzerland \\ 3. University of Toronto, Department of Political Science, Toronto, Canada \\ 4. Technical University of Munich, Bavarian School of Public Policy, Munich, Germany \\ 5. Tohoku University, Graduate School of Environmental Studies, Japan \\ 6. Hiroshima University, Graduate School of International Development and Cooperation, \\ Hiroshima, Japan
}

\begin{abstract}
Phase-out has emerged as a policy approach to confront multiple sustainability crises. From ozone-depleting substances and hazardous chemicals to fossil fuels and transport technologies, phase-out experiences have been documented by diverse scientific communities. To consolidate this dispersed knowledge and inspire more systematic research, we map the evolution of scientific discussions about phase-out through a systematic literature review. Examining 870 papers published since 1970, we trace the evolving nature of phase-out strategies in terms of targets, geographic and industrial contexts, policy instruments and drivers. This provides a multi-faceted overview of an emerging and rapidly growing 'phase-out science' rooted across the full spectrum of scientific enquiry. Evolution of this science is marked by broadening engagement with a growing diversity of targets, contexts, and policies. Our analysis also shows how phase-out policies have recently gained momentum as a tool to tackle climate change, with a particular focus on fossil fuels and associated technologies.
\end{abstract}

\section{Key words}

Phase-out, socio-technical transition, decline policy, technology, sustainability, environment

Word count: 3,630 words (Excluding box, figure captions and references) 


\section{Introduction}

Current systems of production and consumption must undergo deep transformation to achieve sustainable development within planetary boundaries [1]. This fundamental restructuring necessitates the downscaling of specific technologies, materials and practices at the root of various sustainability crises. Strategies to achieve this are attracting increasing interest from scholars and policymakers [2-5]. This rapidly expanding literature recognises that advancing the creation and diffusion of sustainable alternatives is necessary - but not sufficient on its own - for overcoming challenges like climate change, biodiversity loss and chemical pollution [6-8]. Research on complex systems [9, 10] reveals how polluting technologies and wider socio-technical systems are prone to path-dependency and lock-in, which reinforce their persistence and reproduction over long time scales. Explicit policy interventions to rupture these forces are therefore required [11, 12].

This study focuses on a prominent case of such interventions: phase-out. This policy approach actively seeks the sequential termination (i.e. in phases) of a specific technology, substance, or process that causes negative externalities [2]. In the context of sustainable development, its study is merited for two reasons. First, phase-out features heavily in a growing body of scholarship that examines various approaches to hastening the decline of unsustainable socio-technical systems [4, 13-15]. Second, phase-outs lie at the centre of historical and ongoing efforts to mitigate a growing list of sustainability challenges. These include destruction of the ozone layer caused by ozone depleting substances [16]; human and environmental health risks posed by chemicals like mercury, lead and cadmium [17]; and climate change and air pollution caused by fossil fuels and associated technologies like coal-fired power stations [18] and internal combustion engines [19].

Yet, while scientific studies of phase-outs have accumulated for decades [20-23], there has been no systematic effort to comprehensively assess the development and state of this knowledge. Our literature review addresses this gap by mapping the evolution of scientific discussions about phase-out as an approach to tackling environmental sustainability challenges. We trace the inception of the science in 1970 to the present, systematically coding and analysing 870 publications (see Box 1, Methods, and Supplementary Information). Concretely, our multi-faceted analysis captures the evolving character of phase-outs in terms of (i) targets, (ii) drivers, (iii) affected industries, (iv) policy instruments, (v) and geographic context. 
Results reveal the contours of a rapidly growing 'phase-out science' whose character has evolved distinctly over time. Informed by diverse academic disciplines, phase-out science is united by a common interest in the role of this policy approach in tackling various sustainability challenges. Over its 50-year evolution, discussions have broadened to encompass an increasing number and diversity of phase-out targets, geographic and industrial contexts, policy instruments and drivers. The past decade is marked by growing emphasis on using phase-out policies to mitigate climate change, with fossil fuels and associated technologies increasingly targeted.

The contribution of this study is to provide the first global picture of the science and practice of phase-out. We achieve this by conducting the most comprehensive review to date on a decline-related topic, elucidating the career of phase-out within the scientific literature. In parallel, we generate insights on its practical application as a tool for confronting diverse sustainability challenges. In so doing, we help clarify a prominent concept within the growing scholarship about governing the decline of environmentally harmful socio-technical systems [2]. Additionally, by systematically mapping out the core attributes of phase-outs discussed by scientific work accumulated over 50 years, our analysis lays the groundwork for a transdisciplinary research agenda. 


\section{Box 1 Abridged methods}

Following common best practices [24], we employed a systematic mapping review along four steps (see Methods for detailed explanations):

\section{1) Identification and screening of literature}

We iteratively developed a search string, building on previous literature examining the decline of socio-technical systems and their components [2]. We used the Scopus search engine to extract English-language publications, setting conditions to:

- Publication type: Articles and reviews

- Search scope: Title, author keywords and abstract

- Temporal scope: All papers published up to December 31, 2021

- Academic field limitations: None

This returned 1,099 hits. We screened publications for relevance. We included those that explicitly mention phase-out as a strategy for tackling environmental sustainability challenges. We excluded studies discussing phase-out exclusively for reasons unrelated to environmental externalities (e.g. for health, fiscal or national security reasons). This yielded a final corpus of 870 relevant publications (available in Supplementary Data A) after removing duplicates.

\section{2) Analytical frameworks}

We developed research questions relating to five variables: (i) the evolution of phase-out targets, (ii) drivers of phase-outs, (iii) affected industries, (iv) policy instruments, and (v) geographical scope (see Methods for details). To guide the subsequent coding process, we used existing analytical frameworks where available (i.e. industries, policy instruments and geography). For phase-out targets and drivers, we inductively developed new coding frameworks based on the final corpus.

\section{3) Coding procedure}

Abiding by an explicit coding protocol, three experienced researchers systematically coded the thematic content of the portion of abstracts and titles that discuss phase-outs. During the coding process, the analytical frameworks were iteratively adapted to ensure they accurately reflected the coded content. 
4) Visualisation and analysis

We analysed results with the primary purpose of identifying temporal trends. As a secondary goal, we also teased out correlations between each coding category and the most frequently mentioned phase-out targets. 


\section{Findings}

\section{Emergence and evolution of phase-out science}

We identified 870 publications evoking phase-out as an approach to advance sustainability goals. The first appeared in 1970, describing how U.S. electricity utilities addressed chronic air pollution and associated societal pressures by expediting the retirement of coal-fired power plants in favour of nuclear [25]. Then, after accumulating gradually over four decades, research on phase-outs has accelerated rapidly after 2016 (Fig. 1). The last two years are no exception, with 230 studies (26\% of the sample) published in 2020 and 2021 alone.

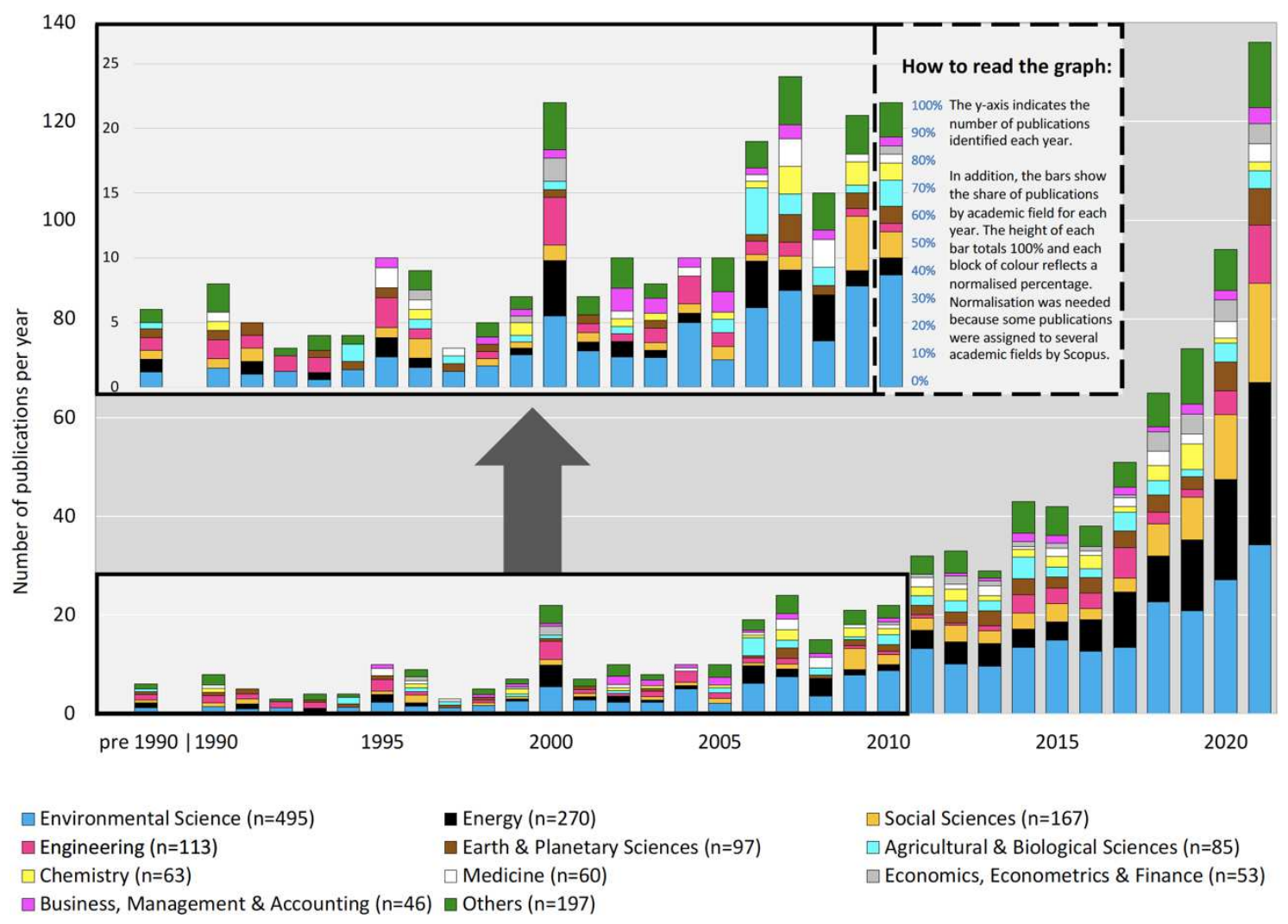

Fig. 1 Number of annual publications and breakdown by academic field

This shows the number of studies published each year $(\mathrm{n}=870)$ that discuss phase-out as an approach to tackling sustainability challenges. Academic fields reflect categories provided by Scopus. For the relative share of each field see Supplementary Data 3 Fig. 1.

A diverse spectrum of academic disciplines has contributed to this body of phase-out science. Furthermore, the relative share of individual disciplines has also evolved. For example, environmental science (shown in blue in Fig. 1) is by far the most prominent 
discipline. Though its engagement with phase-out has expanded over time in absolute terms, its relative share peaked at $39 \%$ in 2010 , then decreasing to $25 \%$ in 2021 (see Supplementary Data 3 Fig. 1). Engineering has peaked even earlier. It accounted for $15 \%$ of papers over 1970-2000 but has since fallen to around 6\% over the last two decades. Recent figures for 2020 and 2021 suggest a renewed interest in phase-out from this discipline, however. In parallel, other fields have consistently strengthened their study of phase-out both in absolute and in relative terms - particularly energy studies and the social sciences.

This disciplinary diversity ensures a myriad of approaches to engaging with phase-out. Overall, we find a tendency in the natural sciences towards empirical work using evidence like environmental indicators to examine the impact of phase-out interventions or advocate for their implementation $[17,21,26]$. Scholarship from the social sciences, business studies and economics is more likely to encompass a theoretical perspective and focus on governance processes or societal drivers and barriers to phase-outs $[2,18]$. This said, a considerable volume of work from the natural sciences $[27,28]$ engages with governance and societal aspects as well.

The study of phase-out has thus evolved into a truly cross-disciplinary enterprise. Though united by a sustained and growing interest in phase-out as an approach to tackle sustainability challenges, in aggregate, this emerging science is yet to coalesce around a coherent or unified set of theories, methods and approaches. 


\section{Targets of phase-out}

Following literature [2], we classified phase-out targets into three broad categories: substances, technologies and processes. Findings reveal a distinct evolution in the targets featured in scientific debates, evidenced by the following three trends.

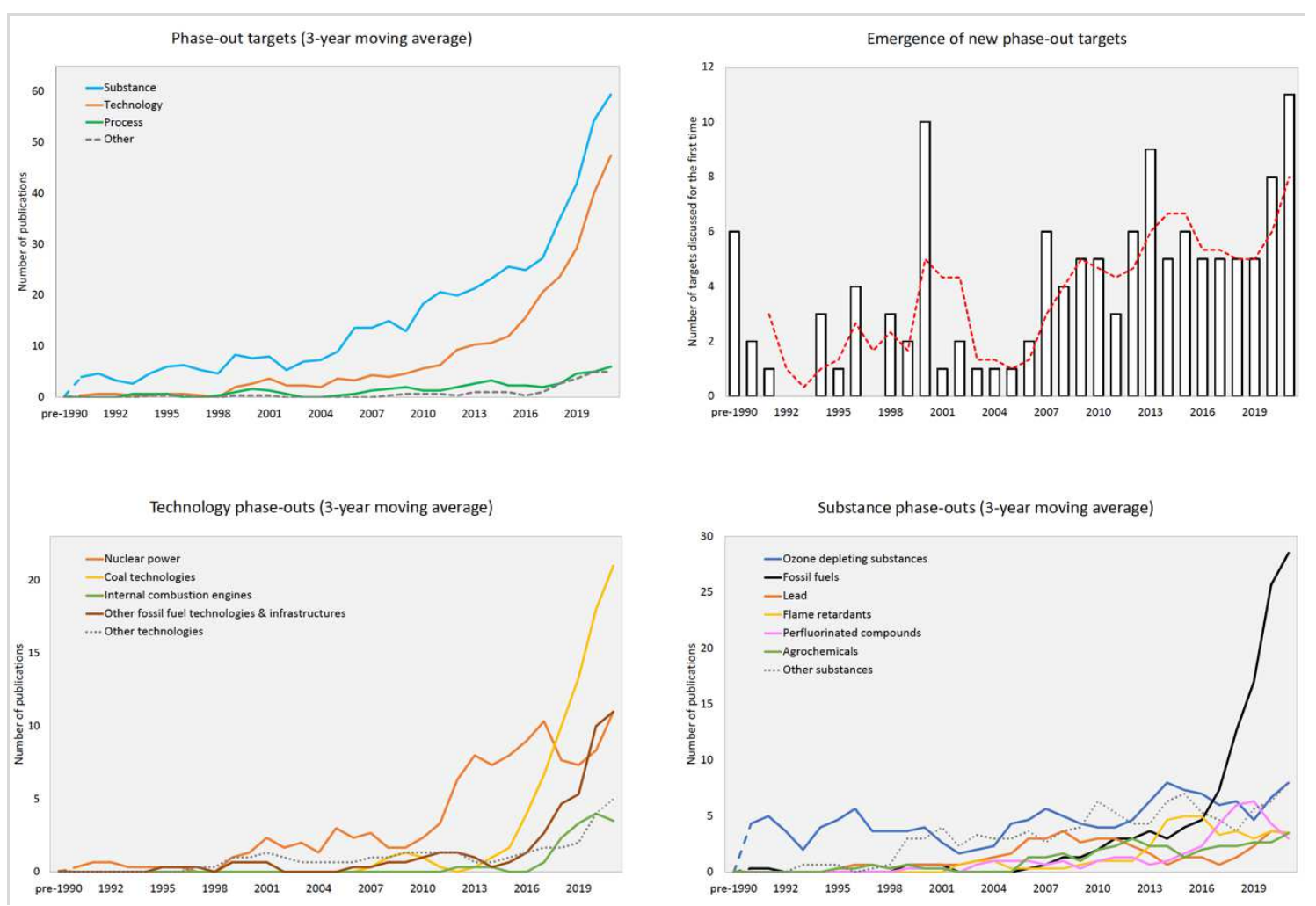

\section{Fig. 2 The evolution of phase-out targets}

The top graphs show (a) elements targeted by the phase-out; and (b) the volume of new phase-out targets each year (i.e. those discussed for the first time in the literature). The red dotted line shows the three-year moving average. The bottom graphs show the number of publications each year that discuss the 10 most recurring targets in the dataset, organised into (c) technologies and (d) substances. These display aggregated coding results for a complete inventory of phase-out targets and those making up at least $5 \%$ of codes for either technology or substances (see Supplementary Data B). All lines show 3-year moving averages.

First, discussions of substances (e.g. flame retardants, lead, agrochemicals) have attracted the most attention (Fig. 2a), representing $61 \%$ of total publications. Also widely discussed are technological targets, comprising $31 \%$ of studies. Phase-outs targeting processes (e.g. fishing, ocean dumping of waste) are evoked infrequently, making up $5 \%$ of papers. In recent years, targets relating to fossil fuels increasingly dominate the literature. These phase-outs either focus on technologies like power plants and internal combustion engines 
or relate to fossil fuels as a substance. In the latter case, phase-out policies target the extraction and use of fossil fuels per se, with prominent approaches being subsidy removal or regulatory instruments.

Second, there is a distinct rise in the volume and diversity of targets discussed over time (Fig. 2b). This reflects increased attention in scientific debates as much as a proliferation of phase-out policies around the world. Specifically, we identified over 120 distinct descriptions of phase-out targets (Supplementary Data B). The diversity of discussed targets is striking, extending well beyond common targets like nuclear and coal to atypical or emerging targets like coloured roofs (which contribute to the heat-island effect) [29], battery cages in poultry farming [30] and plastics [31].

Third, scientific engagement with different phase-out targets has evolved with distinct waves of interest (Fig. 2c-d). These mirror broader and historical developments in society and environmental policy. For technologies, publications on nuclear phase-outs rise and decline in accord with the timing of accidents in Chernobyl in 1986 and Fukushima in 2011. Similarly, real-world events underlie the surge of scientific interest after 2013 in phasing out fossil-fuel technologies and infrastructures - particularly coal. These notably include the Paris Agreement, entering into force in 2016, and the Powering Past Coal Alliance, established the following year to accelerate coal power phase-outs. These same developments influence the substances category. Discussions of fossil fuel phase-outs increase gradually after around 2007 (coinciding with the release of Al Gore's Inconvenient Truth and the IPCC's Fourth Assessment Report) but explode in 2016, again mirroring the adoption of the Paris Agreement. This recent surge of interest is also propelled by recurring commitments within the G7 and G20 to phase-out fossil fuel subsidies in addition to initiatives, especially in Europe, targeting coal mining and oil use in heat and transport systems. Meanwhile, another substance displaying waves of interest is perfluorinated substances. Rising strongly after 2013 and peaking around 2019, this timing reflects the global propagation of phase-out policies and mounting evidence of environmental accumulation and human health impacts of perfluorinated compounds in science [26]. 


\section{Drivers of phase-outs}

Our study indicates that the adoption and implementation of phase-outs are often driven by environmental concerns, such as reducing air or water pollution and wildlife protection. Beyond these, climate change has become a major driving force behind phase-out interventions over the past 15 years (Fig. 3A). This trend continues to intensify. Indeed, $56 \%$ of the studies published in 2020 and 2021 discuss phase-out as decarbonisation strategy. Phase-out has thus entered the mainstream toolkit of climate policy responses.
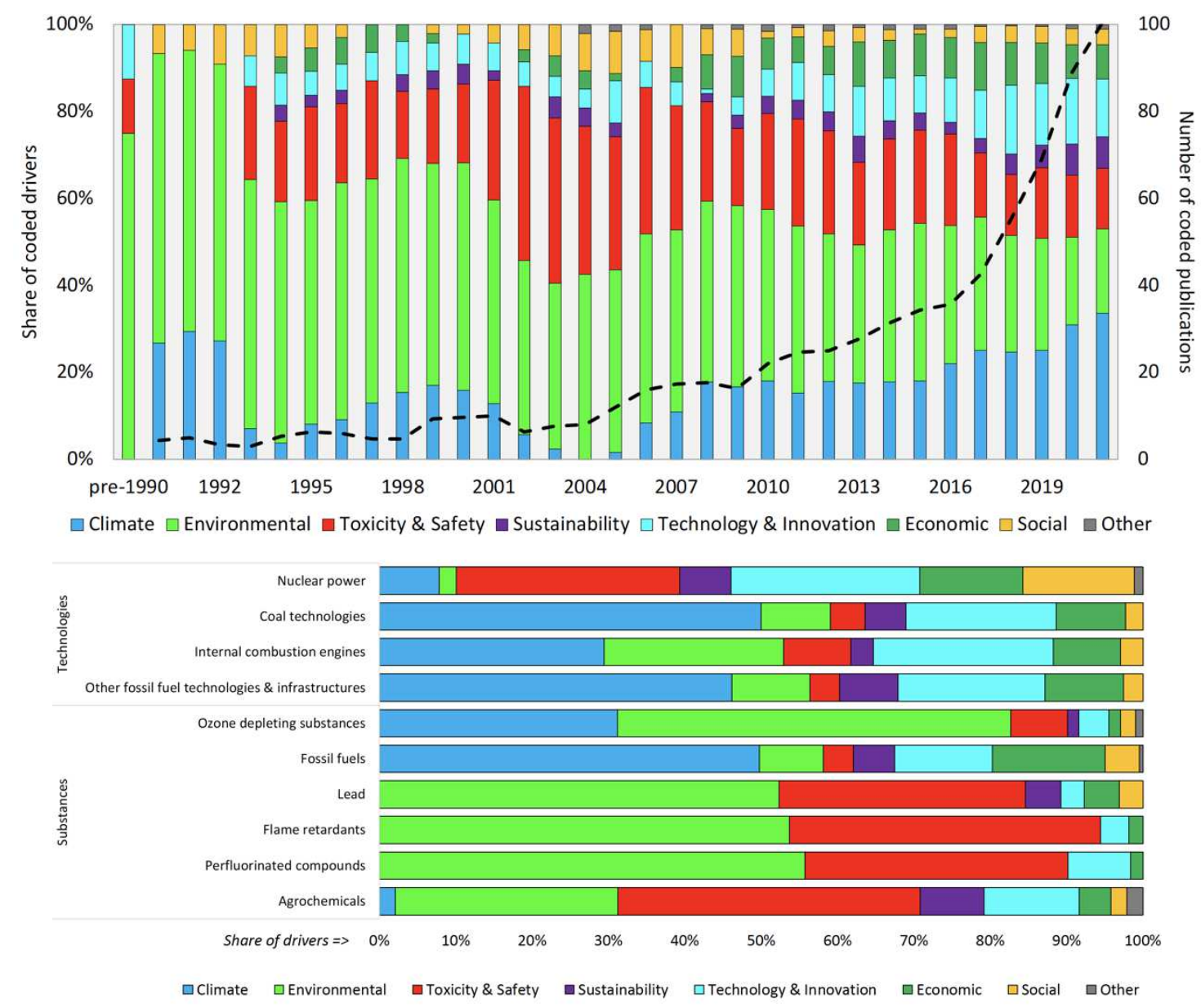

\section{Fig. 3. The evolving nature of phase-out drivers}

(a) shows the relative share of codes for 745 papers mentioning drivers, smoothed with a 3-year average. For reference, the total number of publications receiving a code each year appears as a dotted line, also smoothed to a 3-year running average; (b) shows the relative share of coded drivers for the 10 most frequently mentioned targets. Papers citing multiple drivers received more than one code. Both figures show aggregated results for coding that included more specific driver categories (see Supplementary Data C Fig. 2). 
Diverse co-benefits have emerged alongside environmental concerns as important drivers of phase-outs. These include motivations to advance innovation, economic, social and broader sustainability goals. Of particular note is the role of phase-outs in creating room for innovation processes and the diffusion of alternative arrangements (coded as 'Technology \& innovation'). Some of these discussions emphasise substitution. This involves the replacement or switchover from established to emerging technologies, substances or processes, such as shifting from internal combustion engines to electric drivetrains. Yet other studies conceive phase-outs as a trigger for broader, more systemic change that spans production to consumption. Take, for instance, the use of phase-outs to eliminate harmful chemical substances in manufacturing as a means of promoting a more fundamental shift towards sustainable chemistry [28] or phasing-out single-use plastics to promote a circular economy. This understanding of leveraging phase-out as an innovation driver is gaining momentum, even becoming an emerging subfield in interdisciplinary studies of technology, innovation, and socio-technical systems [4, 14, 15, 32-34].

Economic motivations are also frequently invoked, particularly regarding cost savings and economic growth. For instance, the plummeting cost of renewables along with opportunities to create new businesses and employment has justified phase-outs of fossilfuel power and related subsidies [35]. Meanwhile, phase-outs targeting harmful pesticides and fossil fuel subsidies are pursued to reduce economic burdens posed by health externalities and public expenditures, all while spurring a broader shift towards more sustainable agriculture and a decarbonised economy [36].

We find that the evolving drivers underpinning phase-outs are closely associated with particular targets (Fig. 3b). Two groups of drivers and targets illustrate this.

Enviro-toxic drivers are typically linked to targets such as lead, flame retardants, agrochemicals and perfluorinated compounds. Climate-enviro drivers tend to be associated with fossil-fuel technologies and substances, but also ozone depleting substances. However, some targets resist such classifications. Nuclear phase-out, for instance, is not only associated with interlinked issues of safety, sustainability and societal demands, but also driven by economic and innovation-related considerations [12]. This reflects the technology's tendency to impede the emergence of cheaper, more flexible and diversified renewable energy sources [37]. 


\section{Industry sectors affected by phase-out}

The analysis reveals that a small number of industry sectors dominate discussions about phase-out (see Fig. 4). These include power generation, fossil fuels extraction (including refining and supply) and chemical manufacturing. Power generation is by far the most frequently evoked. This reflects the earlier noted prevalence of studies discussing nuclear phase-outs in addition to the rapidly increasing scholarship on coal and other fossil fuels.

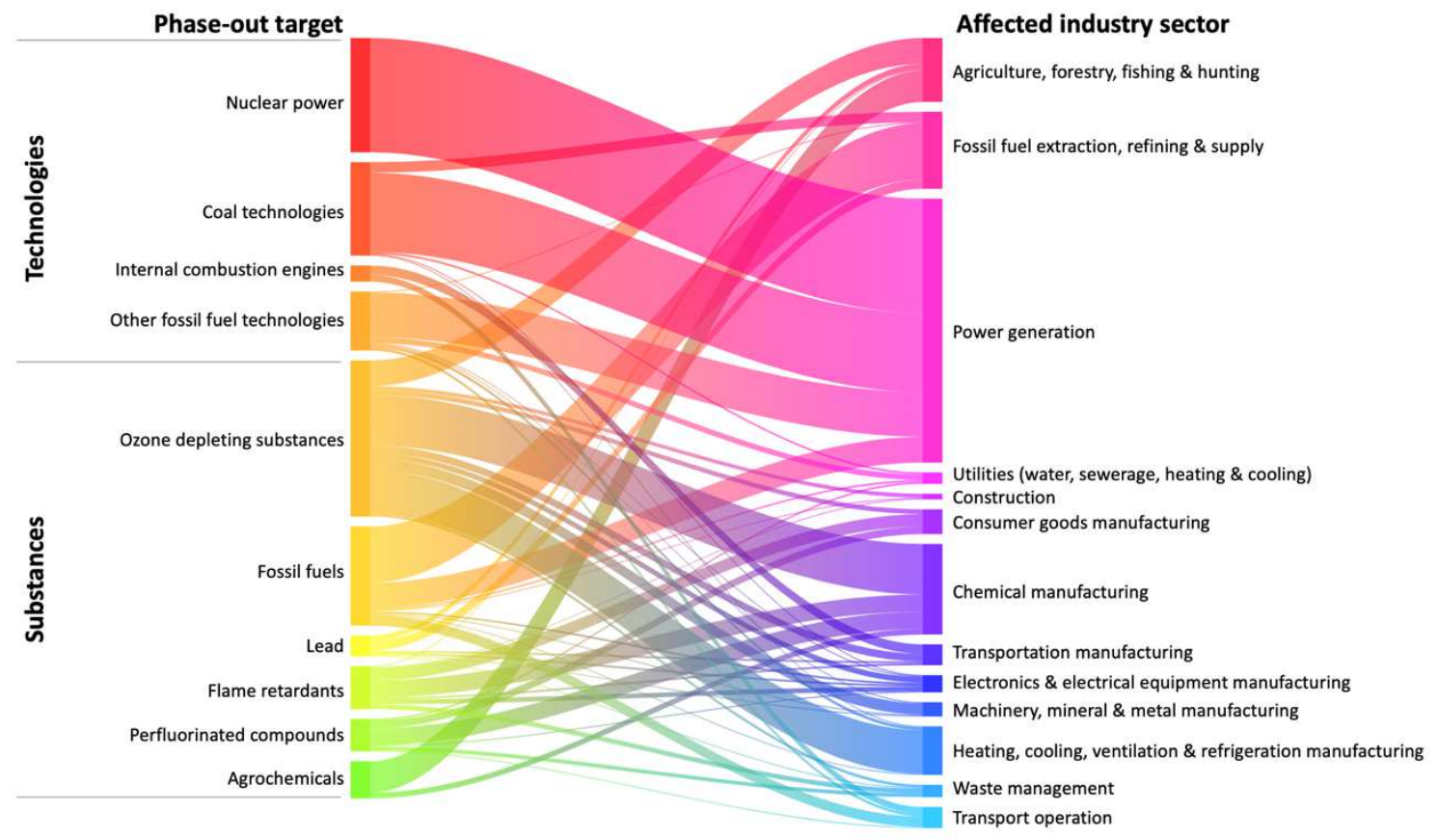

\section{Fig. 4 Industry sectors affected by the most common phase-out targets}

The relative share of industry sectors described in the literature as producing, using or emitting the 10 most frequently mentioned phase-out targets ( $\mathrm{n}=705$ papers). Papers mentioning multiple industries received more than one code.

Results suggest that while some phase-outs will affect a limited number of sectors, others will necessitate the involvement of a much broader range of sectors. For instance, many technology phase-outs (nuclear power, coal technologies, internal combustion engines) tend to affect individual 'mono-industries' such as power generation, fossil fuel extraction or transportation manufacturing. In contrast, substance phase-outs are described as affecting a broader range of sectors. Ozone-depleting substances is one such example, with phase-out regulations targeting numerous and diverse sectors including agriculture (where methyl bromide was widely used as a fungicide), electronics and machinery manufacturing, transportation manufacturing, waste management and construction. Similarly, the scope of 
efforts to reduce human exposure to lead have expanded over time to impact multiple sectors. After initially focusing on leaded gasoline produced by oil refineries and wheel weights produced in transportation manufacturing, recent phase-outs are targeting lead shot used in hunting.

Interestingly, the science gives little indication that a limited number of affected sectors will reduce political and economic barriers to introducing phase-out policies. Rather, those targeting individual industries reliant on the production or use of a specific technology or substance will often confront organised and powerful opposition. Indeed, our dataset contains numerous accounts $[18,19,38-41]$ where the self-preservation interests of nuclear, fossil fuels, agrochemicals and transport manufacturing have delayed, weakened or derailed phase-out policies. Since these sectors are typically large, employing wide-reaching populations, and contributing significantly to local and national economies, resistance can arise equally from workers, the public and politicians.

\section{Phase-out policies}

Two important trends have emerged during the literature's evolution with respect to policies underlying phase-outs. First, mentions of command-and-control instruments dominate our dataset (Fig 5a and Supplementary Data C Fig. 3). These include regulations and restrictions, legally binding treaties and protocols (e.g. the Montreal Protocol for Ozone Depleting Substances) and mandatory environmental standards. Though emphasis on command-and-control declines somewhat after 2000, these instruments are consistently described as the primary policy interventions to pursue phase-outs. Emphasis on command-and-control is especially strong for substances (Fig. 5b). Technology phase-outs are more strongly tied to management and planning instruments, the next most discussed across the dataset. At one end, these instruments reflect managerial approaches like setting phase-out timelines and associated targets (e.g. to reduce production of a substance by a given quantity), reporting mechanisms, and overarching policies that formalise a state's commitment to a phase-out. At the other end, instruments include comprehensive management plans that guide phase-outs by setting priority areas and countermeasures for side-effects like economic disturbances and unemployment. Such plans may rely less on hierarchy and emerge through collaboration across state and industry (Germany's coal phase-out is one example). In sum, the literature's dual emphasis on command-and-control instrument and management and planning approaches portrays phase-out as a process predominantly driven by state intervention. 


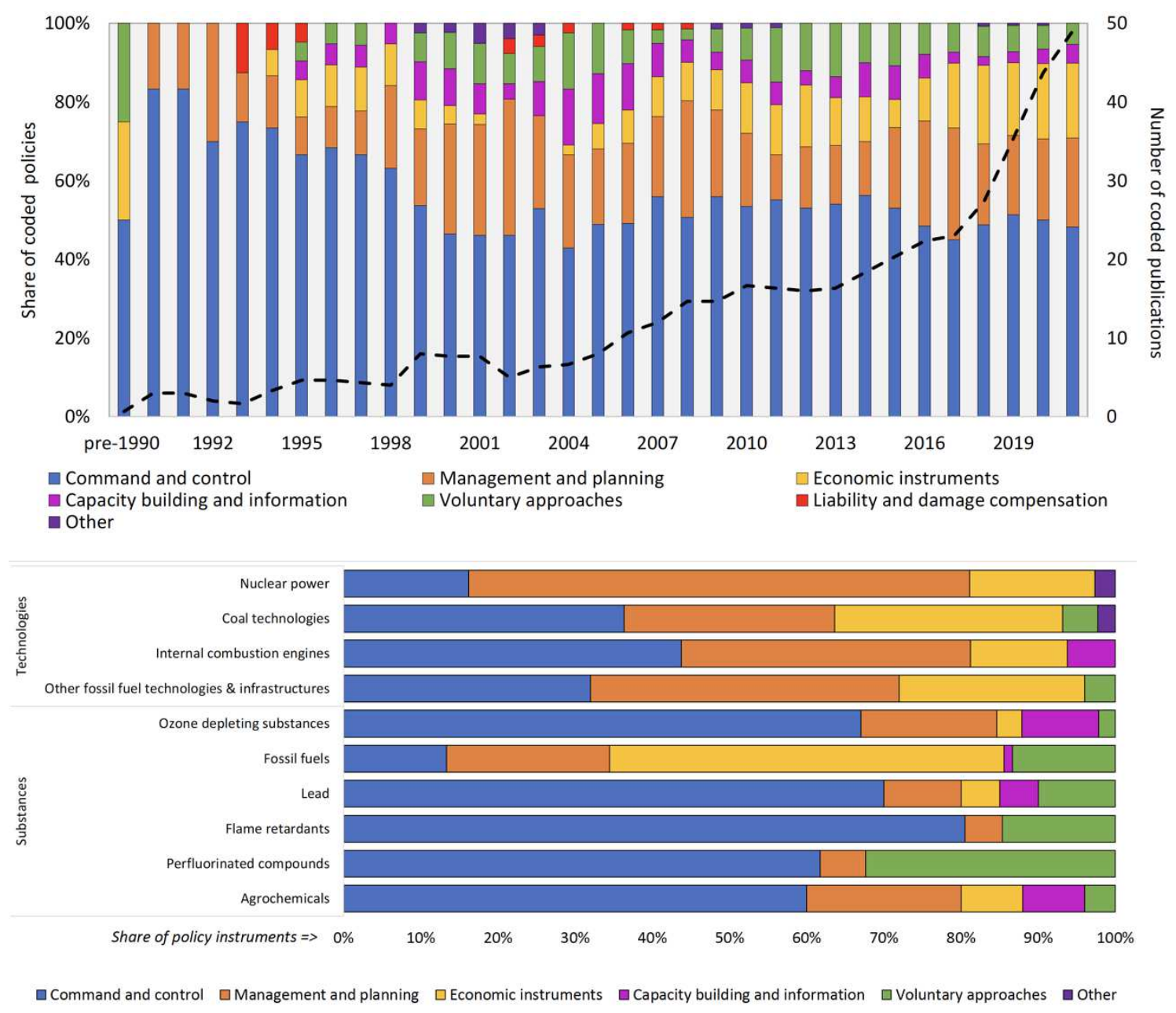

\section{Fig. 5 The evolving nature of phase-out policies}

(a) shows the relative share of codes each year for 443 papers mentioning policies, smoothed with a 3-year average. For reference, the total number of publications receiving a code each year appears as a dotted line, also smoothed to a 3-year running average; (b) shows the relative share of codes for the 10 most frequently mentioned targets. Papers citing multiple policies received more than one code. Policies in both figures show aggregated results for coding that included more specific categories (see Supplementary Data C Fig. 3).

As a second trend, a growing diversity of instruments have been discussed alongside the traditional tools of the state. This reflects a broader tendency to conceptualise and pursue phase-outs as part of mixes rather than single policies. Economic instruments and voluntary approaches are especially discussed. The former consists especially of four types: subsidy reform and removal, pollution pricing (e.g. carbon taxes), financial support (e.g. loans, subsidies) and emissions trading. Of these, subsidy reform and removal are frequently referenced in relation to accelerating the phase-out of fossil fuel extraction and related technologies $[18,36]$. Meanwhile, voluntary approaches tend to be associated 
mostly with substance phase-outs (Fig. 5b); a prominent example being perfluorinated compounds [26]. Typically involving negotiated agreements with industry to expedite substitution with alternatives, voluntary approaches often emerge to fill gaps left by missing or weak regulation [28].

Overall, these trends mirror broader developments within the evolution of environmental policy. Meadowcroft [42] describes how during the 1980s and 1990s, neoliberal approaches to environmental management proliferated as state control was weakened in favour of market-based approaches. Indeed, during the 1990s, our dataset shows a marked decline in mentions of command-and-control approaches while economic instruments and voluntary approaches increased. Nevertheless, in contrast to other spheres of environmental policy (consider, for instance, the hegemony of carbon pricing), our analysis indicates that command-and-control remains the dominant force behind phase-outs.

\section{Geographies of phase-out}

Our findings indicate that phase-out science has diversified its geographic scope of enquiry, extending beyond an early focus on international and North American contexts (Fig. 6a). Prior to 1990 ( $\mathrm{n}=7$ ), reflections on experiences gained from implementing phase-outs in North America (namely the U.S.) and globally (e.g. leaded gasoline and pesticides) dominated the field of inquiry. After this period, scholarship began attending to new geographic terrain, including Europe, Asia and Oceania. Today, all world regions are represented, and the place of emerging economies has expanded considerably. This expanded geographical coverage of literature signifies a global increase in both the study and practice of phase-out. At the same time, studies evoking the global dimension of phase-outs, such as the Stockholm Convention on Persistent Organic Pollutants and other international frameworks, have remained frequent over the past five decades. Besides ozone-depleting substances and fossil fuels (especially subsidies), perfluorinated substances and flame retardants are frequently discussed as targets of global phase-out efforts (Fig. $6 b)$. 

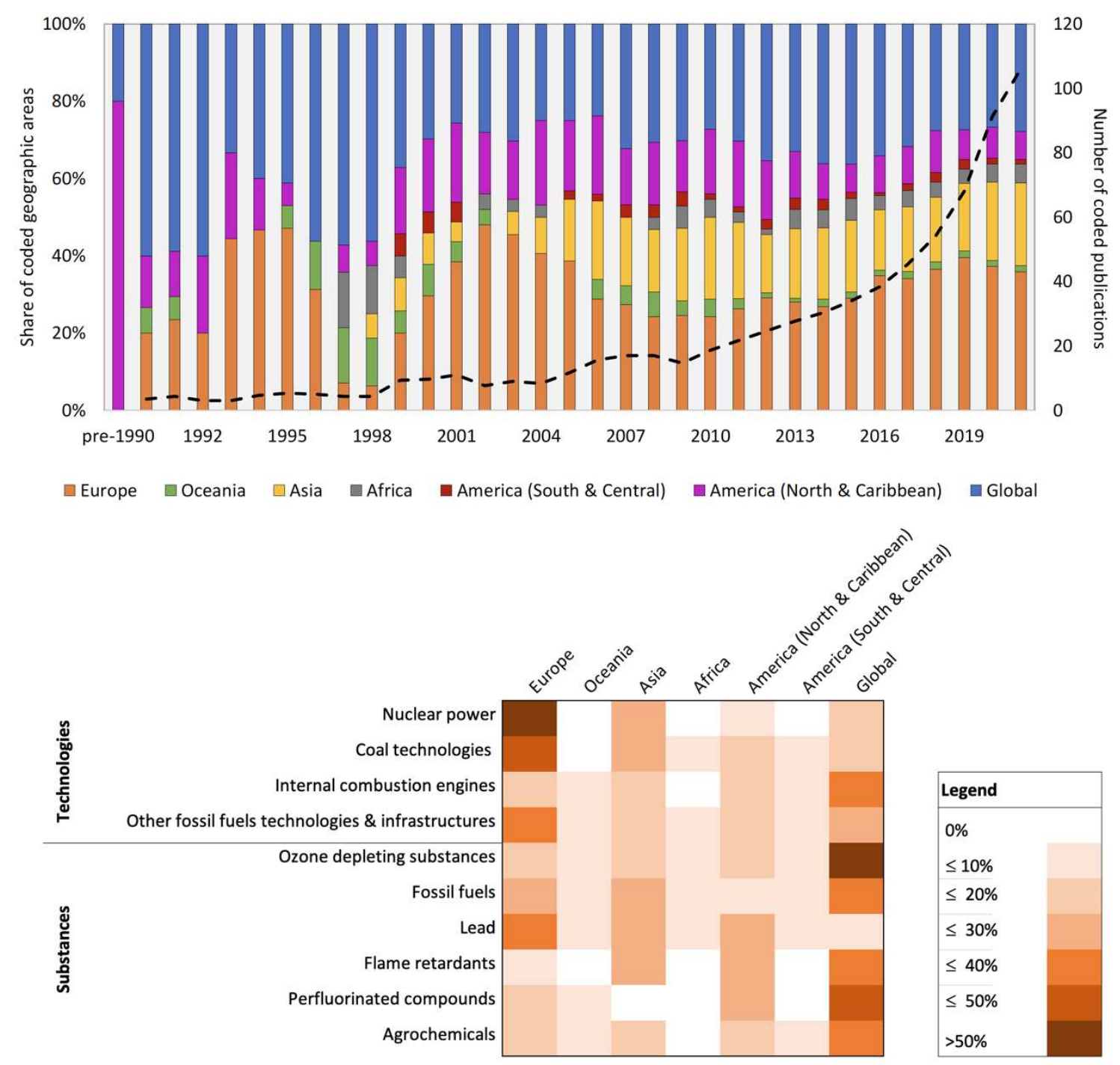

\section{Fig 6. The evolving geographical focus of phase-out discussions}

(a) shows the relative share of codes each year for 743 papers that mentioned a geographical focus, smoothed with a 3-year average. For reference, the total number of publications receiving a code each year appears as a dotted line, also smoothed to a 3-year running average; (b) shows the relative share of codes for the 10 most frequently mentioned targets. Papers citing multiple regions received more than one code. Regions in both figures show aggregated results for more specific coding categories (see Supplementary Data C Fig. 4).

In terms of world regions, the strongest interest is directed at Europe, which features in 30$40 \%$ of publications over the last two decades. The phase-out efforts documented in Europe frequently focus on specific targets (Fig. 6b) including nuclear power, fossil fuels and associated technologies (especially coal power) and lead. The overall prominence of 
European experiences in the literature suggests this region is actively engaged in implementing phase-outs and is seen as a source of instruction for other countries [27, 40].

We find that Asia's relative presence in the dataset expands rapidly after around 2005, becoming the second most discussed region after Europe. This literature covers experiences in both industrialised nations and emerging economies, but concentrates especially on East Asia: particularly China, and to a lesser extent, Japan and Korea. Phaseout efforts in South Asia, namely India, are also discussed. The increased scientific attention to phase-outs targeting Asian countries appears to be a corollary of the accelerating economic development and industrialisation of the region. Driving an increased production and consumption of fossil fuels, chemicals, materials and resources, this growth has triggered externalities like air pollution, environmental contamination and GHG emissions [43]. Phase-out has consequently featured in the various policy responses formulated to tackle such challenges. 


\section{Discussion and conclusions}

This systematic review identified a rapidly growing body of science discussing phase-out as a policy approach. It thereby contributes to rising interest in the decline of unsustainable socio-technical systems and how this process can be managed. Despite differing disciplinary roots and methodological orientations, this nascent phase-out science shares a central interest in how this policy approach can be leveraged to curtail the production and use of substances, technologies and processes at the root of various sustainability challenges. The concept of phase-out thus links diverse scientific communities across engineering, natural sciences, social sciences and beyond, generating important opportunities for interdisciplinary and policy relevant discussions.

Both change and continuity mark the overarching evolution of phase-out science. Evolving from an initial focus on environmental challenges like pollution and ozone depletion, the primary driver of contemporary discussions is climate change. Consequently, fossil fuels and their associated technologies, subsidies and industries are increasingly evoked as phaseout targets. Testifying to a global proliferation of phase-out programmes, the volume and diversity of actual and potential targets invoked by scientists continues to grow. Reflecting this, the geographic scope of studies has expanded beyond an early focus on North America and the global level, with Europe and Asia now featuring heavily in contemporary discussions. Regarding policies, phase-outs are consistently described as chiefly being a process of state intervention, the primary tools being regulation, enforceable agreements and planning. Though increasingly diverse instruments are discussed, in aggregate, the science does not emphasise voluntary approaches as a particularly promising way to phaseout unsustainable substances, technologies and processes.

Documenting over five decades of policymaking experiences, the growing number of targets, drivers, policies and geographies studied in the emerging science indicates that phase-out is far from being a 'new' measure. Rather, its policies build on a multi-decade legacy of experiences, continually adapting to and addressing new challenges. Yet, phaseout is not a silver bullet to all sustainability woes. For example, phase-out efforts have successfully eliminated many first-generation ozone depleting substances, but many have been replaced with climate warming halons, These, in turn, have necessitated a new generation of phase-out programmes. Moreover, phase-out policies can trigger a dash for temporary solutions with short-term benefits but limited long-term prospects for transformative change [5]. Consider for instance the rush to gas driven by coal and nuclear power phase-outs, which risks locking-in a new generation of carbon-intensive 
infrastructure [44]. These historical and ongoing experiences suggest that a holistic understanding of phase-out is required, along with policy designs that embed phase-out goals into agendas for broader sustainability transformations [15]. Such policies should avoid the trap of targeting problematic technologies, substances and processes as individual components, with limited efforts to confront the broader systemic forces that sustain their production and use $[28,45]$.

Though our approach overcomes prior tendencies to focus on small sets of cases, a limitation is that we focused on describing the evolving features of scientific discussions about phase-outs rather than empirical cases of phase-out themselves. Future research could deepen practical understanding by surveying policy trends. Specifically, there is a need to learn from historical and ongoing experiences to identify the conditions that facilitate the successful adoption and accomplishment of phase-outs, particularly amidst resistance from industry and society, and potential economic repercussions like unemployment and stranded assets [46].

Finally, the rapidly expanding and diversifying science examined in this review suggests phase-out will continue as a key tool for tackling sustainability challenges in the future. We thus foresee valuable opportunities for interdisciplinary collaboration across scientific fields to deepen understanding into the process of designing and implementing phase-out interventions, managing affected industries and societal impacts, and evaluating outcomes. Apparently, the attractiveness of phase-out lies in its ability to direct the attention of society, government and industry to 'big bads' in a similar vein to innovation policies that focus on supporting shiny 'new goods'. Yet ideally, phase-out policies should be embedded into a broader mix of sustainability transition policies that seek to fundamentally transform entire systems of unsustainable production and consumption. Otherwise, the effectiveness of phase-out interventions risk being limited to substitution and merely eliminating 'worst offenders'. 


\section{Methods}

This study reviewed the sampled literature using a coding-driven and systematic mapping approach. This allows researchers to systematically process evidence from a large number of publications and to identify trends and patterns. This technique is regarded as more rigorous, objective and replicable than a conventional literature review due to the use of explicit inclusion and exclusion criteria in addition to transparent protocols for identifying thematic trends $[47,48]$. Another feature of the mapping approach is to systematically analyse text data through quantification, where specific themes are identified, coded and then interpreted [49]. Originating from the social sciences, mapping-based reviews have propagated into diverse fields, including sustainability, environmental management and energy literature $[18,50,51]$. We draw methodological guidance and inspiration from all these studies, also following best practices for systematic and scoping reviews in general [24].

\section{Review design, scope and search query}

We firstly established a concrete research aim, which is to understand how scientific literature has described the key features of phase-outs to tackle various sustainability challenges, and how these conceptions have evolved over time. To identify relevant publications for this purpose, we developed a search string (see Supplementary Information Note 1). The string consists of three segments modelled after previous scholarship [2] that conducted a literature review on socio-technical decline. The string was iteratively designed to contain terms that capture: (1) our research topic (i.e. phase-out); (2) diverse mandatory and voluntary policy approaches (e.g. regulation, initiative, plan, incentive), and (3) motivations and context for pursuing phase-out (i.e. environmental, sustainability and climate).

To identify relevant scientific publications, we used the $S$ copus database. This was chosen over other options (e.g. Web of Science, PubMed, Science Direct etc.) due to its wider coverage of journals, abstracts and keywords than competitors [52, 53]. Moreover, Scopus includes a longer temporal coverage, with content records extending back to 1820 . However, since the coverage of $W$ eb of Science is frequently compared to Scopus, we performed a robustness check of our decision to only use the latter. Concretely, we ran our search string on both engines. Although the coverage in terms of publication numbers and academic fields was similar, Web of Science did not generate any hits until 1992. Conversely, Scopus has stronger coverage in early years, the first paper appearing in 1970. Thus, Scopus was deemed better suited to our goal of identifying the origins of the science discussing phase-outs. 
We extracted literature from the Scopus database on August 6, 2021. After sorting, we obtained an initial sample of 825 publications that were subsequently coded and analysed. We repeated the search on January 1 in 2022 to ensure that all studies published during 2021 are contained in our dataset. This procedure brought our final sample to 870 relevant publications.

To capture the diversity of debates and policy experiences across the full disciplinary spectrum of science, we did not set search limitations for academic fields. Furthermore, in applying the criteria for inclusion and exclusion (Box 1), we included publications that explicitly engage with phase-out as a research topic (e.g. those examining phase-out policies, their targets or impacts) as well as publications where phase-out per se is not the principal unit of study. This inclusive approach enabled us to capture a broader spectrum of scientific discussions about phase-out targets and related policies than if limiting our scope only to publications with a strong thematic engagement with phase-out.

\section{Research questions and coding frameworks}

To guide the coding of relevant papers, we developed five sets of associated research questions and coding frameworks (see Supplementary Information Note 3). Coding frameworks include both aggregated parent codes and specific sub-codes. The former provided aggregated descriptions of broad patterns while the latter describe these at a higher resolution. In this study we report mainly the results of parent codes. Guiding research questions and coding frameworks were developed as follows:

1. What is the nature of the phase-out target?

For parent codes, we used categories defined in previous literature (i.e. technology, substance, process) [2] to broadly categorise targets. To more specifically describe the phase-out target, we assigned sub-codes in accord with an inductively created framework (e.g. nuclear power, flame retardants, waste incineration).

2. What drivers are associated with the discussion or implementation of the phase-out?

This used an inductively created framework to categorise both the general driver (e.g. environmental, economic, innovation) and the specific driver (e.g. air pollution, climate change, economic benefits, equity and ethics).

3. What industries are discussed or implied as producing, emitting or using the phase-out target? This used an existing framework, the North American Industry Classification Scheme (NAICS), from the United States Census Bureau [54]. This was used to create parent 
codes to reflect aggregated industry sectors (e.g. chemical manufacturing) and subcodes to reflect specific industries (e.g. sewage treatment facilities). We adapted the NAICS classifications slightly to include industry descriptions not captured by this scheme.

4. What policy instruments are mentioned to achieve the discussed phase-out?

We adopted an established classification framework from the OECD [55] to capture general categories of environmental policies (e.g. command and control, voluntary approaches) as well as specific instruments (e.g. environmental standards, information provision). At the level of instruments, we extended the OECD framework by drawing on diverse descriptions of environmental policies from the governance literature [e.g. 3, $6,56]$. Our coding captured both policies described as actually inducing a phase-out in addition to policies described as desirable, including those under discussion or planning.

5. What geographical region is targeted by the phase-out strategy?

This used an existing framework from the United Nations, the Standard Country or Area Codes for Statistical Use (the so-called '49 Standard') [57], to code geographical regions (e.g. Western Europe, Northern Africa) and specific countries.

\section{Coding procedure}

Coding was guided by a detailed coding protocol that was developed and shared by all coders. This consisted of an iteratively designed and integrated package of: (i) overarching principles, (ii) explicit descriptions of coding options provided by the above coding frameworks, and (iii) specific rules to guide their operationalisation (see Supplementary Information Note 3). More concretely, one of our overarching principles was to code only the portion of abstracts that explicitly connect to the discussed phase-out target. Another high-level principle was to design coding frameworks that permitted coding at the finest level of detail possible during initial coding (i.e. without having to aggregate detailed descriptions). Meanwhile, to ensure consistency across coding decisions and preserve the internal validity of data, we recorded and shared decisions about particular coding variables across the research team. For instance, we fixed a specific rule that mentions of phase-outs targeting coal extraction should be coded as a substance rather than a process. This decision is underpinned by the logic that the goal of such policies is to reduce the production and consumption of a fossil fuel substance as opposed to seeking to eliminate resource extraction per se.

The initial coding frameworks and protocol were adapted iteratively during a pilot phase. 
This involved coded approximately $20 \%$ of all publications contained in the final corpus and adapting our coding protocol described above to ensure replicability and accuracy. During this phase, we also probed the extent to which the surveyed literature is actually suited to answering our research questions. This subsequently provoked the reformulation of two of our research questions to ensure a tighter match with the nature of our dataset.

To minimise subjective bias in coding and support a consistent and replicable outcome of the coding process, three experienced researchers took charge of manually coding the titles, abstracts and author keywords of all publications that form the final corpus. Full papers were only consulted in the case of ambiguous language in the title or abstract. To ensure that only information relevant to the discussed phase-out was coded, we omitted from all coding the portion of the abstract, title or keywords unrelated to this topic. Finally, multiple codes were assigned to papers as needed - e.g., a publication discussing several drivers accordingly received several codes for drivers.

The three coders sequentially coded all publications. The first coder was responsible for the initial screening for relevance and for highlighting relevant text portions and suggesting parent codes. The second (lead) coder verified these decisions and independently coded each publication according to all five coding categories. The third coder reviewed all coding decisions. When discrepancies were encountered, these were discussed among the coders until a solution was reached. Finally, a random sample of the final corpus was crosschecked once again at the end of the coding process by two coders to detect and remove remaining inconsistencies.

\section{Data availability}

The bibliographic details (i.e. year of publication, author, title, author keywords, abstract and journal title) for the sample of relevant publications included for analysis are available in Supplementary Data A.

\section{Code availability}

Comprehensive information on the complete coding strategy used to code papers is available in Supplementary Information Note 3. Our full coding results are available as Supplementary Data B. 


\section{List of supplementary material}

\section{Supplementary Information - Search string and coding protocol}

This PDF file contains three supplementary notes to explain the procedure for: 1)

Developing the search string; 2) Screening relevant publications; and 3) Coding protocol.

\section{Supplementary Data A - Literature sample and codebook}

This Excel file contains three sheets containing: 1) the full sample of coded literature, 2) the coding results, 3) the code book explaining the coding system.

\section{Supplementary Data B - Inventory of phase-out targets}

This Excel file contains three sheets containing: 1) Inventory of all coded phase-out targets,

2) aggregated categories to identify top-10 targets, 3) classification of industries

\section{Supplementary Data C- Additional figures}

This PDF file contains supplementary figures 1, 2, 3 and 4. 


\section{References}

1. O’Neill, D.W., et al., A good life for all within planetary boundaries. Nature Sustainability, 2018. 1(2): p. 88-95.

2. Rosenbloom, D. and A. Rinscheid, Deliberate decline: An emerging frontier for the study and practice of decarbonization. WIREs Climate Change, 2020. e669(n/a).

3. Kivimaa, P., et al., Moving beyond disruptive innovation: A review of disruption in sustainability transitions. Environmental Innovation and Societal Transitions, 2021. 38: p. 110-126.

4. Normann, H.E., Conditions for the deliberate destabilisation of established industries: Lessons from U.S. tobacco control policy and the closure of Dutch coal mines. Environmental Innovation and Societal Transitions, 2019. 33: p. 102-114.

5. Markard, J. and D. Rosenbloom, A tale of two crises: COVID-19 and climate. Sustainability: Science, Practice and Policy, 2020. 16(1): p. 53-60.

6. Kivimaa, P. and F. Kern, Creative destruction or mere niche support? Innovation policy mixes for sustainability transitions. Research Policy, 2016. 45(1): p. 205-217.

7. David, M., Moving beyond the heuristic of creative destruction: Targeting exnovation with policy mixes for energy transitions. Energy Research \& Social Science, 2017. 33: p. 138-146.

8. Hoffmann, S., J. Weyer, and J. Longen, Discontinuation of the automobility regime? An integrated approach to multi-level governance. Transportation Research Part A: Policy and Practice, 2017. 103: p. 391-408.

9. Unruh, G.C., Understanding carbon lock-in. Energy Policy, 2000. 28(12): p. 817-830.

10. Seto, K.C., et al., Carbon Lock-In: Types, Causes, and Policy Implications. Annual Review of Environment and Resources, 2016. 41(1): p. 425-452.

11. Geels, F.W., et al., The Socio-Technical Dynamics of Low-Carbon Transitions. Joule, 2017. 1(3): p. 463-479.

12. Rogge, K.S. and P. Johnstone, Exploring the role of phase-out policies for low-carbon energy transitions: The case of the German Energiewende. Energy Research \& Social Science, 2017. 33: p. 128-137.

13. Koretsky, Z. and H. van Lente, Technology phase-out as unravelling of socio-technical configurations: Cloud seeding case. Environmental Innovation and Societal Transitions, 2020. 37: p. 302-317.

14. David, M. and N. Schulte-Römer, Phasing out and in: System transition through disassociation in the German energy transition - The case of light and coal. Energy Research \& Social Science, 2021. 80: p. 102204.

15. Rinscheid, A., et al., From terminating to transforming: The role of phase-out in sustainability transitions. Environmental Innovation and Societal Transitions, 2021. 41: p. 27-31. 
16. Solomon, S., J. Alcamo, and A.R. Ravishankara, Unfinished business after five decades of ozone-layer science and policy. Nature Communications, 2020. 11(1): p. 4272.

17. You, M., Interpretation of the source-specific substantive control measures of the Minamata Convention on Mercury. Environment International, 2015. 75: p. 1-10.

18. Diluiso, F., et al., Coal transitions — part 1: a systematic map and review of case study learnings from regional, national, and local coal phase-out experiences. Environmental Research Letters, 2021. 16(11): p. 113003.

19. Meckling, J. and J. Nahm, The politics of technology bans: Industrial policy competition and green goals for the auto industry. Energy Policy, 2019. 126: p. 470-479.

20. Glynn, S., Constructing a selection environment: competing expectations for CFC alternatives. Research Policy, 2002. 31(6): p. 935-946.

21. von Storch, H., et al., Four decades of gasoline lead emissions and control policies in Europe: a retrospective assessment. Science of The Total Environment, 2003. 311(1): p. 151-176.

22. van der Voet, E., et al., Cadmium in the European Community: a Policy-Oriented Analysis. Waste Management \& Research, 1994. 12(6): p. 507-526.

23. Gençsü, I., et al., Phasing out public financial flows to fossil fuel production in Europe. Climate Policy, 2020. 20(8): p. 1010-1023.

24. Haddaway, N.R. and B. Macura, The role of reporting standards in producing robust literature reviews. Nature Climate Change, 2018. 8(6): p. 444-447.

25. Friedlander, G.D., Power, pollution, and the imperiled environment. IEEE Spectrum, 1970. 7(11): p. 40-50.

26. Land, M., et al., What is the effect of phasing out long-chain per- and polyfluoroalkyl substances on the concentrations of perfluoroalkyl acids and their precursors in the environment? A systematic review. Environmental Evidence, 2018. 7(1): p. 4.

27. Kanstrup, N., Lessons learned from 33 years of lead shot regulation in Denmark. Ambio, 2019. 48(9): p. 999-1008.

28. Tickner, J., K. Geiser, and M. Coffin, The U.S. Experience in Promoting Sustainable Chemistry (9 pp). Environmental Science and Pollution Research, 2005. 12(2): p. 115123.

29. Sproul, J., et al., Economic comparison of white, green, and black flat roofs in the United States. Energy and Buildings, 2014. 71: p. 20-27.

30. Hartcher, K.M. and B. Jones, The welfare of layer hens in cage and cage-free housing systems. World's Poultry Science Journal, 2017. 73(4): p. 767-782.

31. Anagnosti, L., et al., Worldwide actions against plastic pollution from microbeads and microplastics in cosmetics focusing on European policies. Has the issue been handled effectively? Marine Pollution Bulletin, 2021. 162: p. 111883. 
32. Rentier, G., H. Lelieveldt, and G.J. Kramer, V arieties of coal-fired power phase-out across Europe. Energy Policy, 2019. 132: p. 620-632.

33. Vögele, S., et al., Transformation pathways of phasing out coal-fired power plants in Germany. Energy, Sustainability and Society, 2018. 8(1): p. 25.

34. van Oers, L., et al., The politics of deliberate destabilisation for sustainability transitions. Environmental Innovation and Societal Transitions, 2021. 40: p. 159-171.

35. Monasterolo, I. and M. Raberto, The impact of phasing out fossil fuel subsidies on the lowcarbon transition. Energy Policy, 2019. 124: p. 355-370.

36. Matsuo, T. and T.S. Schmidt, Hybridiring low-carbon technology deployment policy and fossil fuel subsidy reform: a climate finance perspective. Environmental Research Letters, 2017. 12(1): p. 014002.

37. Carrara, S., Reactor ageing and phase-out policies: global and regional prospects for nuclear power generation. Energy Policy, 2020. 147: p. 111834.

38. Trencher, G., et al., Discursive resistance to phasing out coal-fired electricity: Narratives in Japan's coal regime. Energy Policy, 2019. 132: p. 782-796.

39. Kungl, G. and F.W. Geels, Sequence and alignment of external pressures in industry destabilisation: Understanding the downfall of incumbent utilities in the German energy transition (1998-2015). Environmental Innovation and Societal Transitions, 2018. 26: p. 78100.

40. Donley, N., The USA lags behind other agricultural nations in banning harmful pesticides. Environmental Health, 2019. 18(1): p. 44.

41. Steckel, J.C. and M. Jakob, The political economy of coal: Lessons learnt from 15 country case studies. World Development Perspectives, 2021. 24: p. 100368.

42. Meadowcroft, J., Greening the state?, in Comparative Environmental Politics

Theory, Practice, and Prospects, P.F. Steinberg and S.D. VanDeveer, Editors. 2012, The MIT Press. p. 63-87.

43. Yang, L., et al., Environmental-social-economic footprints of consumption and trade in the AsiaPacific region. Nature Communications, 2020. 11(1): p. 4490.

44. Brauers, H., I. Braunger, and J. Jewell, Liquefied natural gas expansion plans in Germany: The risk of gas lock-in under energy transitions. Energy Research \& Social Science, 2021. 76: p. 102059.

45. Thorpe, B. and M. Rossi, Require Safer Substitutes and Solutions: Making the Substitution Principle the Cornerstone of Sustainable Chemical Policies. NEW SOLUTIONS: A Journal of Environmental and Occupational Health Policy, 2007. 17(3): p. 177-192.

46. Koretsky, Z., Phasing out an embedded technology: Insights from banning the incandescent light bulb in Europe. Energy Research \& Social Science, 2021. 82: p. 102310. 
47. Haddaway, N.R., et al., The benefits of systematic mapping to evidence-based environmental management. Ambio, 2016. 45(5): p. 613-620.

48. O'Leary, B.C., et al., Evidence maps and evidence gaps: evidence review mapping as a method for collating and appraising evidence reviews to inform research and policy. Environmental Evidence, 2017. 6(1): p. 19.

49. Hsieh, H.-F. and S.E. Shannon, Three Approaches to Qualitative Content Analysis. Qualitative Health Research, 2005. 15(9): p. 1277-1288.

50. Fisch-Romito, V., et al., Systematic map of the literature on carbon lock-in induced by longlived capital. Environmental Research Letters, 2021. 16(5): p. 053004.

51. McKinnon, M.C., et al., What are the effects of nature conservation on buman well-being? $A$ systematic map of empirical evidence from developing countries. Environmental Evidence, 2016. 5(1): p. 8.

52. Falagas, M.E., et al., Comparison of PubMed, Scopus, Web of Science, and Google Scholar: strengths and weaknesses. The FASEB Journal, 2008. 22(2): p. 338-342.

53. Peñasco, C., L.D. Anadón, and E. Verdolini, Systematic review of the outcomes and tradeoffs of ten types of decarbonization policy instruments. Nature Climate Change, 2021. 11(3): p. 257-265.

54. United States Census Bureau. North American Industry Classification Scheme. 2017 [cited 2017 December 30]; Available from: https://www.census.gov/naics/.

55. OECD, Sustainable Development: Critical Issues. 2001, Paris: OECD Publishing.

56. Hood, C. Summing up the parts: Combining Policy Instruments for Least-Cost Climate Mitigation Strategies. 2011 [cited 2021 July 30]; Available from: https://www.thepmr.org/content/summing-parts-combining-policy-instrumentsleast-cost-climate-mitigation-strategies-iea-20-0.

57. Statistics Division of the United Nations Secretariat. Standard Country or Area Codes for Statistical Use (49 Standard). 2021 [cited 2021 December 30]; Available from: https://unstats.un.org/unsd/methodology/m49/. 


\section{Supplementary Files}

This is a list of supplementary files associated with this preprint. Click to download.

- SupplementaryInformationSearchstringandcodingprotocol.pdf

- SupplementaryDataALiteraturesampleandcodebook.xlsx

- SupplementaryDataBInventoryofphaseouttargets.xlsx

- SupplementaryDataCAdditionalfigures.pdf 